\title{
Honey bee survival is affected by interactions between field-relevant rates of fungicides and insecticides used in apple and blueberry production
}

\author{
Paul Manning ${ }^{a \star}$, Krilen Ramanaidu ${ }^{b}$, and G. Christopher Cutler \\ ${ }^{a}$ Department of Plant, Food and Environmental Sciences, Faculty of Agriculture, Dalhousie University, \\ Truro, NS B2N 5E3, Canada; ' Nurture Atlantic, P.O. Box 29115, Moncton, NB E1G 2R5, Canada \\ *paul.manning@dal.ca
}

\begin{abstract}
Certain compounds of low toxicity can increase the susceptibility of an organism to toxic substances; this is known as potentiation. Demethylation inhibiting (DMI) fungicides can potentiate insecticides by impairing the production of detoxification enzymes. As both DMI fungicides and insecticides can be used near or during crop bloom, the combination may be hazardous if exposed to pollinators. Using pesticides used in blueberry or apple production, we conducted laboratory bioassays to test how combinations of field-relevant concentrations of DMI fungicides and insecticides affected honey bee (Apis mellifera) survival. We found propiconazole, a DMI fungicide, potentiated the toxicity of the neonicotinoid insecticide acetamiprid. We found no evidence of propiconazole potentiating field-relevant concentrations of the spinosyn insecticide spinetoram. We also found that the DMI fungicide flusilazole potentiated spinetoram but not acetamiprid. A fungicidal formulation combining pyraclostrobin and boscalid did not potentiate either insecticide. Given that bees can be simultaneously exposed to multiple pesticides, understanding the potential of pesticide potentiation and synergism may help mitigate risks associated with pollinator exposure to pesticides.
\end{abstract}

Cutler GC. 2017. Honey bee survival is affected by interactions between fieldrelevant rates of fungicides and insecticides used in apple and blueberry production. FACETS 2: 910-918. doi:10.1139/ facets-2017-0025

Editor: Peter G. Kevan

Received: March 13, 2017

Accepted: July 26, 2017

Published: November 20, 2017

Corrected: May 10, 2018

Copyright: (C) 2017 Manning et al. This work is licensed under a Creative Commons Attribution 4.0 International License (CC BY 4.0), which permits unrestricted use, distribution, and reproduction in any medium, provided the original author(s) and source are credited.

Published by: Canadian Science Publishing

\section{Introduction}

Farmers often use several pesticides for managing different pests and diseases. This means insects may be exposed to multiple compounds in the field. Multi-compound exposure may be simultaneous or successive, with different potential outcomes. In most cases, there is no interaction between pesticides in a mixture (Cedergreen 2014) and effects of combined substances tend to be additive, such that the total effect is equal to the sum of responses of the components of the mixture. In some cases, antagonistic responses are observed, whereby one pesticide interferes with another to produce an overall response that is less than the sum response. Related to this are synergistic responses, where all substances in the mixture are toxic to an organism, but the combined response is greater than the sum of the component parts. Different still is potentiation, where substances of low toxicity enhance the susceptibility of an organism to toxic substances (Barile 2013).

Pesticide potentiation has been identified between insecticides and demethylation inhibiting (DMI) fungicides, also referred to as ergosterol-biosynthesis-inhibiting fungicides (Iwasa et al. 2004). 
DMI fungicides obtain their fungicidal activity through disrupting biosynthesis of ergosterol, the dominant lipid in fungal cell membranes (Köller and Scheinpflug 1987). Although ergosterol does not occur in animal cells, exposing insects to DMI fungicides inhibits production of cytochrome P450 enzymes (Pillings and Jepson 1993). When production of these enzymes is inhibited, insects are unable to efficiently metabolize toxic compounds and consequently their susceptibility to insecticide exposure may increase up to more than 1000-fold (Iwasa et al. 2004).

Potentiation of insecticides by DMI fungicides has been widely observed in honey bees (Apis mellifera L.) (Pillings and Jepson 1993; Iwasa et al. 2004; Thompson et al. 2014). Determining if these interactions occur at field-relevant concentrations of widely used pesticides is important; failure to appreciate potential interactions of pesticides could result in toxic exposure to honey bees and other non-target insects (Desneux et al. 2007). At the same time, failure to manage pests and diseases with pesticides during critical times for pollination could lead to significant economic losses for farmers (Melathopoulos et al. 2014).

We conducted laboratory experiments to examine the potential of DMI fungicides to potentiate insecticides in honey bees. Using field rate concentrations, we tested five different pesticides, two insecticides, and three fungicides used in apple and lowbush blueberry production. We predicted that both DMI fungicides would potentiate the activity of insecticides. We predicted a third fungicidal product that operates via a different mode of action would not interact with either of the insecticides.

\section{Materials and methods}

\section{Pesticides and exposure concentrations}

We selected five pesticides that are used in blueberry or apple production. The chosen pesticides can be used during or near the time when honey bees pollinate. We tested two insecticidal and three fungicidal products, each having a different active ingredient (Table 1). We used two insecticides: the neonicotinoid acetamiprid (Assail ${ }^{\circledR} 70 \mathrm{WP}$, Mississauga, Ontario, Canada), an agonist of nicotinic acetylcholine receptors (nAChR); and spinetoram (Delegate ${ }^{\circledR}$ WG, Dow AgroSciences Canada, Calgary, Alberta, Canada), a spinosyn allosteric modulator acting at the macrocyclic lactone site of non-desensitizing nAChRs. We tested three fungicides for their ability to potentiate the activity of acetamiprid and spinetoram. Two were DMI fungicides-propiconazole (Topas ${ }^{\circledR} 250 \mathrm{E}$, Syngenta Canada, Guelph, Ontario, Canada) and flusilazole (Nustar ${ }^{\circledR}$, E.I. Dupont, Mississauga, Ontario, Canada) - and the third was pyraclostrobin + boscalid (Pristine ${ }^{\circledR}$ WG, BASF, Mississauga, Ontario, Canada), a fungicide containing two active ingredients, each of which works through inhibiting different components of electron chain transport in fungal mitochondria (Karadimos et al. 2005).

Table 1. Pesticides and their test concentrations used in honey bee acute toxicity experiments examining potentiation of insecticides by fungicides.

\begin{tabular}{lccc}
\hline Product & Active ingredient & Field rate $(\mathrm{ppm})^{a}$ & Test concentration $\left.^{a} \mathrm{ppm}\right)$ \\
\hline Assail $^{\circledR} 70 \mathrm{WP}$ & Acetamiprid & 560 & 560 \\
Delegate $^{\circledR}$ & Spinetoram & 256 & 25 \\
Topas $^{\circledR}$ & Propiconazole & 613 & 613 \\
Nustar $^{\circledR}$ & Flusilazole & 12 & 12 \\
Pristine $^{\circledR}$ & Pyraclostrobin + boscalid & 2432 & 2432 \\
\hline
\end{tabular}

${ }^{a}$ Field rate corresponds to the concentration of the active ingredient(s) when diluted according to the manufacturer's instructions. 
Products were diluted in distilled water from commercially available formulations (Table 1). All compounds were tested at a field rate, with the exception of spinetoram, which was diluted below the typical tank-mix concentration. Field rates of this insecticide are lethally toxic to honey bees (Shimokawatoko et al. 2012), bumble bees (Gradish et al. 2012b), and solitary bees (Gradish et al. 2012a) by acute oral and contact exposure. Spray of spinetoram is not permitted when honey bees are foraging, but bees could nonetheless be exposed to this insecticide post-spray. We used a concentration of $25 \mathrm{ppm}$, approximately one-tenth of the tank concentration. For potentiation experiments, both products were combined within the same solution. For example, the solution applied to test spinetoram potentiation by flusilazole had a combined exposure concentration of $25 \mathrm{ppm}$ spinetoram and $12 \mathrm{ppm}$ flusilazole.

\section{Toxicity assays}

The experiments were conducted in late summer. We collected honey bees from hives at the Dalhousie University Agricultural Campus approximately $1 \mathrm{~h}$ prior to starting the experiments. All bees used in the experiments were foraging workers returning to the hive. Bees were aspirated into $1 \mathrm{~L}$ mason jars at the hive entrance using a modified hand vacuum (Oliver 2008). Once approximately $50-60$ bees were aspirated, the jar was closed by affixing a lid fitted with a $1 \mathrm{~mm}$ screen, which allowed ventilation. Two cotton wicks soaked in 1:1 sucrose-distilled water solution in the jar provided sustenance and moisture. Six jars of bees were collected this way for each experiment. In the laboratory, bees were anesthetized by allowing carbon dioxide to diffuse into each Mason jar for $30 \mathrm{~s}$, and then bees were randomly placed into groups of 9-11 individuals. Each group of anesthetized bees was considered an experimental replicate. While still anaesthetized, bees were treated on their dorsal side with $1 \mu \mathrm{L}$ of treatment solution (Table 1) using a PAX 100-3 micro-applicator (Burkard Scientific, Uxbridge, UK). Bees in the control treatment were exposed to $1 \mu \mathrm{L}$ of distilled water.

Bees were held in inverted $355 \mathrm{~mL}$ clear plastic cups with $\sim 1 \mathrm{~mm}$ diameter perforations for ventilation (Evans et al. 2009). The inverted cup was placed on the lid of a $90 \mathrm{~mm}$ Petri dish. A hole was cut at the top to insert a drip feeder, which consisted of an inverted $25 \mathrm{~mL}$ glass vial of 1:1 sucrose:distilled water solution capped with a plastic lid. Two small holes were made in the cap so that when the feeder was inverted the sugar solution could be freely accessed by the bees. A $10 \mathrm{~cm} \times 10 \mathrm{~cm}$ square of incontinency underpad lined the Petri dish to absorb occasional droplets from the drip feeder. All replicates from different treatments were held in the same growth cabinet at $25{ }^{\circ} \mathrm{C}$ and $60 \%$ relative humidity.

We conducted three experiments. The first examined if the test concentrations of propiconazole or pyraclostrobin + boscalid would potentiate the activity of acetamiprid; each treatment was replicated $n=5$ times. The second experiment tested if these same concentrations of fungicides would potentiate the insecticidal activity of spinetoram; each treatment was replicated $n=4$ times. Following the results with propiconazole, we conducted a third experiment with another DMI fungicide, in which we tested if flusilazole would potentiate the activity of acetamiprid and spinetoram; each treatment was replicated $n=5$ times. Honey bee mortality at $48 \mathrm{~h}$ following exposure was used as a dependent variable throughout (Laurino et al. 2011). To assess mortality, each motionless bee was prodded with a blunt probe. Any bee that remained fully motionless or stationary with occasional twitching was considered dead. Final sample sizes varied from $N=24$ to $N=30$.

\section{Analysis}

To test the effect of pesticide exposure on honey bee survival, we first fitted generalized linear models with binomial error distributions that predicted mortality as a function of pesticide exposure. Model 
diagnostics showed that in all cases, data were overdispersed; thus all final models instead used a quasi-binomial error structure (Crawley 2012). Through an $F$ test, we compared each of these models to a null model that included only the intercept. In all cases, the $p$-value generated from the $F$ test indicated statistical significance $(p \leq 0.05)$. Through examining residual deviance, for all models we found that including pesticide exposure better explained variation in honey bee mortality than an intercept alone. When pesticide treatment was found to significantly affect survival, we compared the difference among treatment means using Tukey's honest significant differences test. All analyses were conducted in R (R Core Team 2016). We compared means using the multcomp package (Hothorn et al. 2008), and produced figures using ggplot2 (Wickham 2009). Experimental data and accompanying $\mathrm{R}$ code are available in Supplementary Materials 1 and 2, respectively.

\section{Results}

In the acetamiprid experiment, we found that pesticide exposure significantly influenced honey bee survival $\left(F_{5,22}=15.59, p<0.001\right)$. Post hoc comparison of the means showed that the field-relevant concentration of propiconazole potentiated the toxicity of acetamiprid causing $100 \%$ mortality (Fig. 1). None of the other treatments differed significantly from the control (Fig. 1).

In the spinetoram experiment, we found no effect of pesticide exposure on honey bee survival $\left(F_{5,18}=1.17, p=0.36\right)$. Mortality was low across all treatments, whether pesticides were applied alone or in combination (Fig. 2).

In the flusilazole experiment, we found that pesticide exposure significantly influenced honey bee survival $\left(F_{5,24}=4.30, p=0.006\right)$. Flusilazole potentiated spinetoram causing high levels of honey bee mortality that were significantly greater than the control, flusilazole, and spinetoram treatments (Fig. 3).

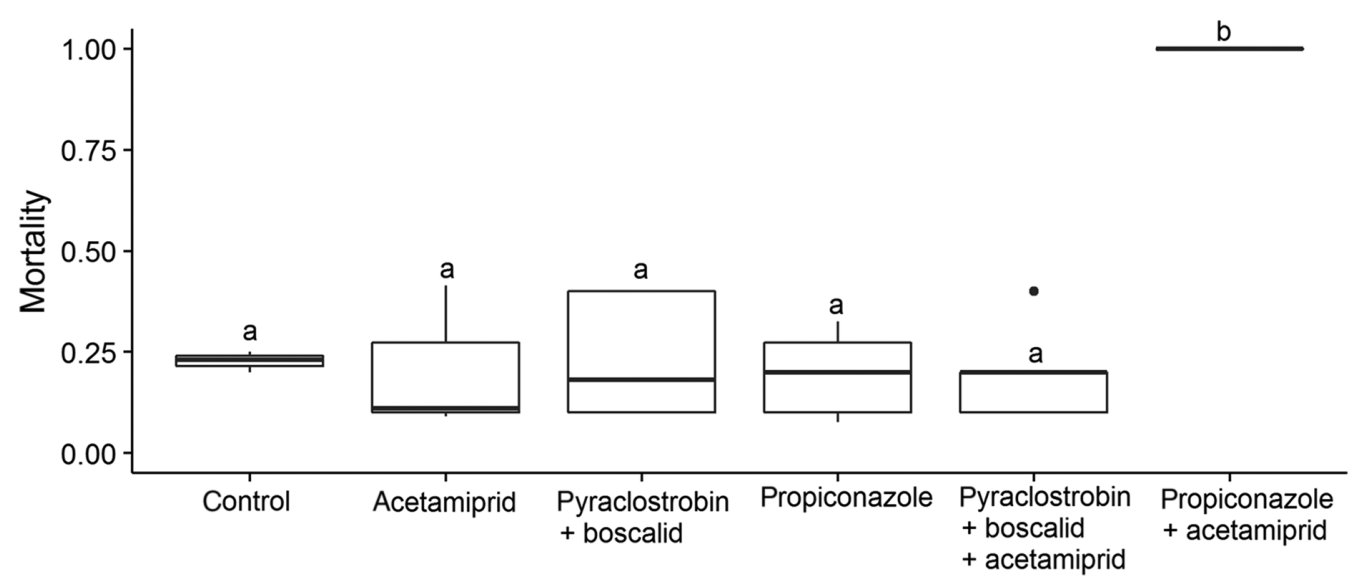

Fig. 1. Box and whisker plot showing honey bee worker mortality at $48 \mathrm{~h}$ following exposure to a $1 \mu \mathrm{L}$ dose of the insecticide acetamiprid, the fungicide boscalid + pyraclostrobin, the fungicide propiconazole, or a combination of acetamiprid with either fungicide. The lower and upper limits of the box represent the inter quartile range (IQR) of the data. The horizontal line within each box represents the median. Whiskers extend from the boxes to show maximum and minimum values; if this distance is $>1.5 \times$ the IQR, observations appear as a filled point. The means of treatments sharing the same letter do not differ significantly from each other (Tukey's honest significant differences test, $\alpha=0.05)$. All treatments were replicated in quintuplicate $(n=5)$, but two control replicates were lost when bees escaped $(N=28)$. 


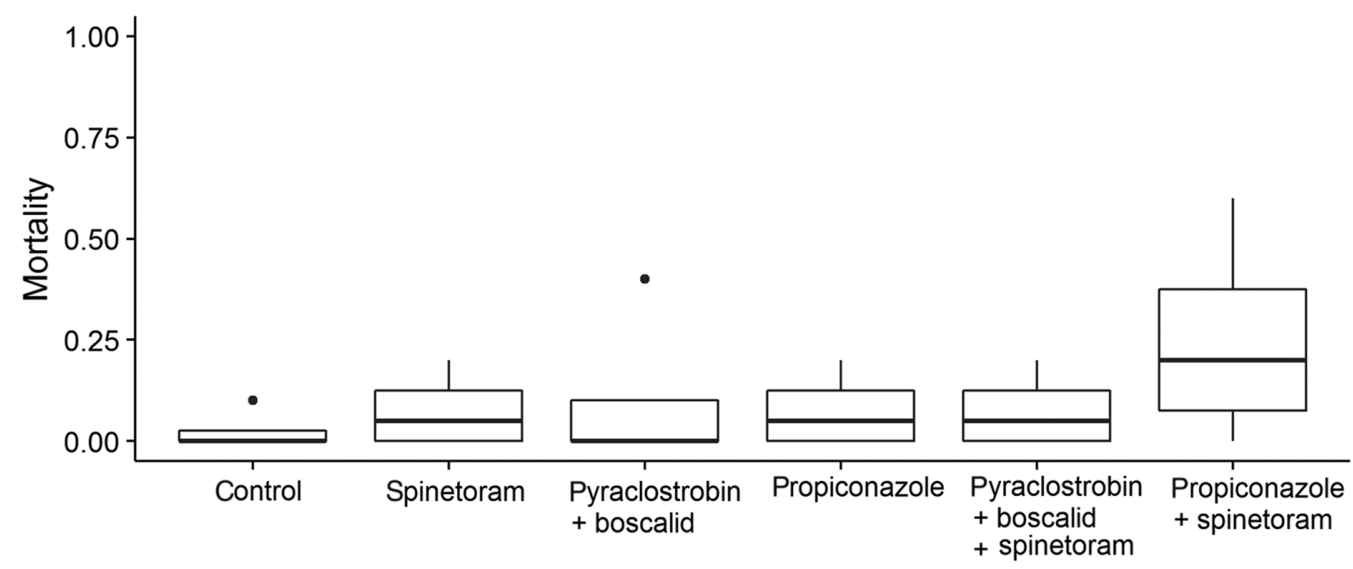

Fig. 2. Box and whisker plot showing honey bee worker mortality at $48 \mathrm{~h}$ following exposure to a $1 \mu \mathrm{L}$ dose of the insecticide spinetoram, the fungicide boscalid + pyraclostrobin, the fungicide propiconazole, or a combination of spinetoram with either fungicide. The lower and upper limits of the box represent the inter quartile range (IQR) of the data. The horizontal line within each box represents the median. Whiskers extend from the boxes to show maximum and minimum values; if this distance is $>1.5 \times$ the IQR, observations appear as a filled point. The meeans of treatments do not differ significantly from each other. All treatments were replicated in quadruplicate $(n=4)$ for a total $N=24$.

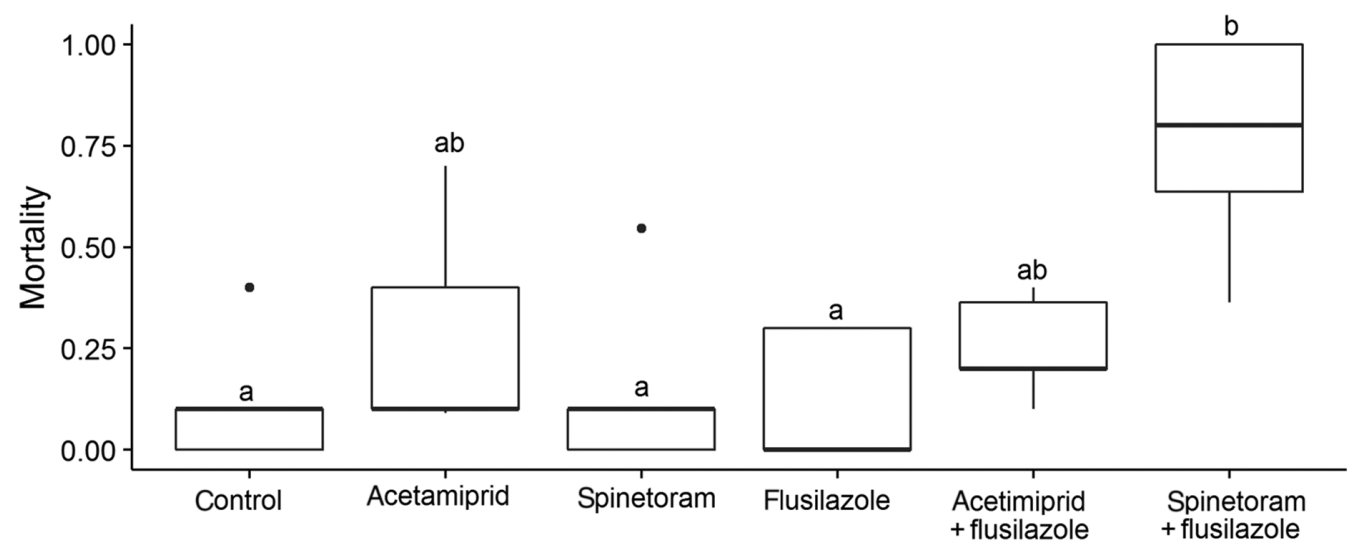

Fig. 3. Box and whisker plot showing honey bee worker mortality at $48 \mathrm{~h}$ following exposure to a $1 \mu \mathrm{L}$ dose of the fungicide flusilazole, the insecticide acetamiprid, the insecticide spinetoram, or a combination of flusilazole with either insecticide. The lower and upper limits of the box represent the inter quartile range (IQR) of the data. The horizontal line within each box represents the median. Whiskers extend from the boxes to show maximum and minimum values; if this distance is $>1.5 \times$ the IQR, observations appear as a filled point. The means of treatments sharing the same letter do not differ significantly from each other (Tukey's honest significant differences test, $\alpha=0.05)$. All treatments were replicated in quintuplicate $(n=5)$ for a total $N=30$.

\section{Discussion and conclusions}

In contrast to our first prediction, we found that DMI fungicides did not potentiate the activity of insecticides in all cases. The nature of the interaction was dependent on the compounds used in combination. We found that the DMI fungicide propiconazole potentiated acetamiprid, but not spinetoram, whereas the DMI fungicide flusilazole potentiated spinetoram, but not acetamiprid. Similar variability in the presence or strength of interactions between DMI fungicides and insecticides has 
been documented in other studies. Pillings and Jepson (1993) found that field rates of 10 different DMI fungicides enhanced the contact toxicity of the pyrethroid lamda-cyhalothrin between 1.4- and 15-fold. Thompson et al. (2014) tested all possible combinations between four neonicotinoids and four DMI fungicides on contact exposure and found that only a single combination caused potentiation. Knowledge of combinations of certain DMI fungicides and insecticides that do not interact could be useful in reducing risks to non-target invertebrates while simultaneously managing pest and disease damage within tolerable levels.

In the field, insecticides and fungicides are typically applied in "medium-sized" droplets, which are 226-325 $\mu \mathrm{m}$ in diameter (Grisso et al. 2013). A $1 \mu \mathrm{L}$ droplet as used in our experiments has an equivalent volume of $1 \mathrm{~mm}^{3}$. A sphere with this volume would have a diameter of about $1240 \mu \mathrm{m}$, approximately 3.8- to 5.5 -fold the size of a typical pesticide droplet. When considered in terms of volume, a bee in our laboratory experiment would encounter the equivalent of between 55 and 166 mediumsized pesticide droplets. If honey bees were flying within the vicinity of a blueberry field or apple orchard during pesticide application, one could speculate this level of exposure could occur. We found that field rates of propiconazole potentiated acetamiprid, causing 100\% mortality. Although growers sometimes apply acetamiprid during bloom if a pest threshold is exceeded, honey bees should fortunately not be simultaneously exposed to these pesticides because propiconazole and acetamiprid are applied pre- and post-bloom, respectively (Assail ${ }^{\circledR}$ Insecticide Product Label, E.I. DuPont Co., Mississauga, Ontario L5M 2J4, Canada; Topas ${ }^{\circledR}$ Insecticide Product Label, Syngenta Canada, Guelph, Ontario N1G 4Z3, Canada). Although these compounds have been detected in pollen sampled from honey bee hives (Mullin et al. 2010), exposure was 3-4 orders of magnitude lower than the tank-rate concentrations we tested here.

The observed potentiation between flusilazole and spinetoram in our experiments may be of concern in some field situations. Topical exposure to $25 \mathrm{ppm}$ spinetoram, a concentration well below that of a typical tank-mix concentration, caused low mortality in our experiments $(11.6 \% \pm 5.5 \%$, mean $\pm \mathrm{SE})$. Field-rate exposure to flusilazole also caused little mortality $(12.0 \% \pm 6.6 \%)$. However, combined exposure to flusilazole and spinetoram caused six-fold more mortality in honey bees $(76 \% \pm 10.7 \%)$, suggesting the potential for potentiation between these compounds, even when exposure concentrations of spinetoram are low. Spinetoram apparently degrades relatively quickly under field conditions, with a half-life of only $2.6 \mathrm{~d}$ (Malhat 2013), but can nevertheless remain effective for extended periods. For example, foliar applications at recommended field rates caused $100 \%$ mortality of larval Choristoneura rosaceana (Lepidoptera: Tortricidae) up to $59 \mathrm{~d}$ post-application (Sial et al. 2011). Although flusilazole degrades relatively rapidly in the field (Yu et al. 2011), it has been shown to potentiate multiple insecticides (Thompson et al. 2014) and has been detected in pollen (David et al. 2016), honey bees, and honey (Lambert et al. 2013). Moreover, flusilazole can be applied to crops like apple during full flower (Nustar ${ }^{\circledR}$ Fungicide Product Label, E.I. Dupont, Mississauga, Ontario, Canada), with spinetoram applied to apple for insect pest control just before full bloom. This suggests that honey bees could concurrently be exposed to concentrations of flusilazole and spinetoram similar to those used in our experiment.

As predicted, the fungicide pyraclostrobin + boscalid did not interact with either of the two insecticides tested. Neither pyraclostrobin nor boscalid interfere with cytochrome P450s or other detoxification enzymes in insects, making potentiation of insecticides by this fungicide highly unlikely.

It is important to note that although simultaneous exposure to DMI fungicides and insecticides may occur in the field and potentiation may occur, reported incidences of such interactions in the field are rare (Cedergreen 2014). For example, Schmuck et al. (2003) identified potentiation in laboratory assays but failed to observe any effect on lethal and sublethal metrics in semi-field studies. Despite the risk pesticides pose to pollinating insects, they remain a valuable tool for optimizing agricultural production. 
Although pollination services are critical for pollinator limited crops (Gallai et al. 2009), the benefits of pollinators may go unrealized in the absence of insect and disease management (Melathopoulos et al. 2014). Identifying pesticides that pose limited risk to pollinators is important in maintaining economic and environmentally sustainable production. Given that pollinators are frequently exposed to numerous compounds in the field (Mullin et al. 2010), understanding the potential of pesticide potentiation and synergisms can help mitigate risk associated with pollinator exposure to pesticides.

\section{Acknowledgements}

This research was supported by the NSERC-Canadian Pollination Initiative (CANPOLIN), and a Killam Postdoctoral Fellowship to PM. This is publication no. 146 of NSERC-CANPOLIN.

\section{Author contributions}

KR and GCC conceived and designed the study. PM and KR performed the experiments/collected the data. PM, KR, and GCC analyzed and interpreted the data. GCC contributed resources. PM, KR, and GCC drafted or revised the manuscript.

\section{Competing interests}

The authors have declared that no competing interests exist.

\section{Data accessibility statement}

All relevant data are within the paper and in the Supplementary Material.

\section{Supplementary material}

The following Supplementary Material is available with the article through the journal website at doi:10.1139/facets-2017-0025.

Supplementary Material 1

Supplementary Material 2

\section{References}

Barile FA. 2013. Principles of toxicology testing. 2nd edition. CRC Press, Boca Raton, Florida. 603 p.

Cedergreen N. 2014. Quantifying synergy: a systematic review of mixture toxicity studies within environmental toxicology. PLoS ONE, 9: e96580. PMID: 24794244 DOI: 10.1371/journal. pone.0096580

Crawley MJ. 2012. The R book. 2nd edition. John Wiley \& Sons, Chichester, UK. 1080 p.

David A, Botías C, Abdul-Sada A, Nicholls E, Rotheray EL, Hill EM, et al. 2016. Widespread contamination of wildflower and bee-collected pollen with complex mixtures of neonicotinoids and fungicides commonly applied to crops. Environment International, 88: 169-178. PMID: 26760714 DOI: 10.1016/j.envint.2015.12.011

Desneux N, Decourtye A, and Delpuech J-M. 2007. The sublethal effects of pesticides on beneficial arthropods. Annual Review of Entomology, 52: 81-106. PMID: 16842032 DOI: 10.1146/annurev. ento.52.110405.091440 
Evans JD, Chen YP, di Prisco G, Pettis J, and Williams V. 2009. Bee cups: single-use cages for honey bee experiments. Journal of Apicultural Research, 48: 300-302. DOI: 10.1080/00218839.2009. 11101548

Gallai N, Salles J-M, Settele J, and Vaissière BE. 2009. Economic valuation of the vulnerability of world agriculture confronted with pollinator decline. Ecological Economics, 68: 810-821. DOI: 10.1016/j. ecolecon.2008.06.014

Gradish AE, Scott-Dupree CD, and Cutler GC. 2012a. Susceptibility of Megachile rotundata to insecticides used in wild blueberry production in Atlantic Canada. Journal of Pest Science, 85: 133-140. DOI: $10.1007 /$ s10340-011-0391-0

Gradish AE, Scott-Dupree CD, Frewin AJ, and Cutler GC. 2012b. Lethal and sublethal effects of some insecticides recommended for wild blueberry on the pollinator Bombus impatiens. The Canadian Entomologist, 144: 478-486. DOI: 10.4039/tce.2012.40

Grisso R, Hipkins P, Askew S, Hipkins L, and Mccall D. 2013. Nozzles: selection and sizing. Virginia Cooperative Extension, Blacksburg, Virginia. 12 p. [online]: Available from pubs.ext.vt.edu/content/ dam/pubs_ext_vt_edu/442/442-032/442-032_pdf.pdf.

Hothorn T, Bretz F, and Westfall P. 2008. Simultaneous inference in general parametric models. Biometrical Journal, 50: 346-363. PMID: 18481363 DOI: 10.1002/bimj.200810425

Iwasa T, Motoyama N, Ambrose JT, and Roe RM. 2004. Mechanism for the differential toxicity of neonicotinoid insecticides in the honey bee, Apis mellifera. Crop Protection, 23: 371-378. DOI: $10.1016 /$ j.cropro.2003.08.018

Karadimos DA, Karaoglanidis GS, and Tzavella-Klonari K. 2005. Biological activity and physical modes of action of the $\mathrm{Q}_{\mathrm{o}}$ inhibitor fungicides trifloxystrobin and pyraclostrobin against Cercospora beticola. Crop Protection, 24: 23-29. DOI: 10.1016/j.cropro.2004.06.004

Köller W, and Scheinpflug H. 1987. Fungal resistance to sterol biosynthesis inhibitors: a new challenge. Plant Disease, 71: 1066-1074. DOI: 10.1094/PD-71-1066

Lambert O, Piroux M, Puyo S, Thorin C, L'Hostis M, Wiest L, et al. 2013. Widespread occurrence of chemical residues in beehive matrices from apiaries located in different landscapes of western France. PLoS ONE, 8: e67007. PMID: 23799139 DOI: 10.1371/journal.pone.0067007

Laurino D, Porporato M, and Patetta A. 2011. Toxicity of neonicotinoid insecticides to honey bees: laboratory tests. Bulletin of Insectology, 64: 107-113.

Malhat FM. 2013. Simultaneous determination of spinetoram residues in tomato by high performance liquid chromatography combined with QuEChERS method. Bulletin of Environmental Contamination and Toxicology, 90: 222-226. PMID: 23229295 DOI: $10.1007 /$ s00128-012-0885-3

Melathopoulos AP, Tyedmers P, and Cutler GC. 2014. Contextualising pollination benefits: effect of insecticide and fungicide use on fruit set and weight from bee pollination in lowbush blueberry. Annals of Applied Biology, 165: 387-394. DOI: 10.1111/aab.12143

Mullin C, Frazier M, Frazier JL, Ashcraft S, Simonds R, vanEngelsdorp D, et al. 2010. High levels of miticides and agrochemicals in North American apiaries: implications for honey bee health. PLoS ONE, 5: e9754. PMID: 20333298 DOI: 10.1371/journal.pone.0009754 
Oliver R. 2008. Suck-a-bee. American Bee Journal, 148: 719-721.

Pillings ED, and Jepson DC. 1993. Synergism between EBI fungicides and a pyrethroid insecticide in the honeybee (Apis mellifera). Pesticide Management Science, 39: 293-297. DOI: 10.1002/ ps. 2780390407

R Core Team. 2016. R: a language and environment for statistical computing. Vienna, Austria [online]: Available from $r$-project.org/.

Schmuck R, Stadler T, and Schmidt H-W. 2003. Field relevance of a synergistic effect observed in the laboratory between an EBI fungicide and a chloronicotinyl insecticide in the honeybee (Apis mellifera L, Hymenoptera). Pest Management Science, 59: 279-286. PMID: 12639044 DOI: $10.1002 /$ ps.626

Shimokawatoko Y, Sato N, Yamaguchi Y, and Tanaka H. 2012. Development of the novel insecticide spinetoram $\left(\right.$ Diana $\left.^{\circledR}\right)$. Research and Development Report, Sumitomo Kagaku, Tokyo, Japan. 14 p.

Sial AA, Brunner JF, and Garczynski SF. 2011. Biochemical characterization of chlorantraniliprole and spinetoram resistance in laboratory-selected obliquebanded leafroller, Choristoneura rosaceana (Harris) (Lepidoptera: Tortricidae). Pesticide Biochemistry and Physiology, 99: 274-279. DOI: $10.1016 / j$.pestbp.2011.01.006

Thompson HM, Fryday SL, Harkin S, and Milner S. 2014. Potential impacts of synergism in honeybees (Apis mellifera) of exposure to neonicotinoids and sprayed fungicides in crops. Apidologie, 45: 545-553. DOI: 10.1007/s13592-014-0273-6

Wickham H. 2009. ggplot2: elegant graphics for data analysis. Springer, New York, New York [online]: Available from ggplot2.org/.

Yu S, Qin D, Wu Q, Guo X, Han L, and Jiang S. 2011. Residue and dissipation dynamics of flusilazole in apple and soil. Bulletin of Environmental Contamination and Toxicology, 86(3): 319-322. PMID: 21290102 DOI: 10.1007/s00128-011-0203-5 